\title{
Demonstrator \\ V2V Communications in Automotive Multi-sensor Multi-target Tracking
}

Matthias Röckl, Thomas Strang, Matthias Kranz

Matthias.Roeckl@dlr.de, Thomas.Strang@dlr.de, Matthias.Kranz@dlr.de

\section{German Aerospace Center (DLR)}

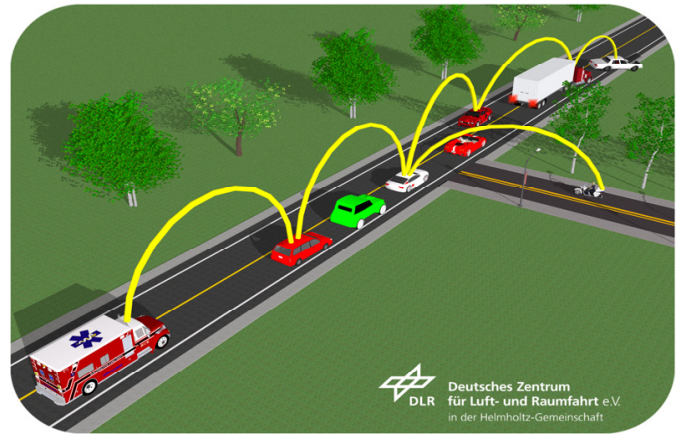

\section{Vehicle-2-Vehicle Communications}

$>$ Wireless communications between vehicles (inter-vehicle communication) and between vehicles and infrastructure

$>$ Exchange of orthogonal, complementary or redundant information over short-medium range exceeding line-ofsight horizon

7 Use cases: Traffic Jam Warning, Cooperative Collision Avoidance, Cooperative Adaptive Cruise Control, ...

\section{Cooperative Object Detection And Ranging (CODAR)}

7 Dynamic probabilistic situation estimation

7 Flexible and scalable sensor incorporation

7 Integration of V2V communication as virtual sensor

7 Service-oriented architecture (completely OSGI based)

$>$ Plug'n'Play vehicle deployment

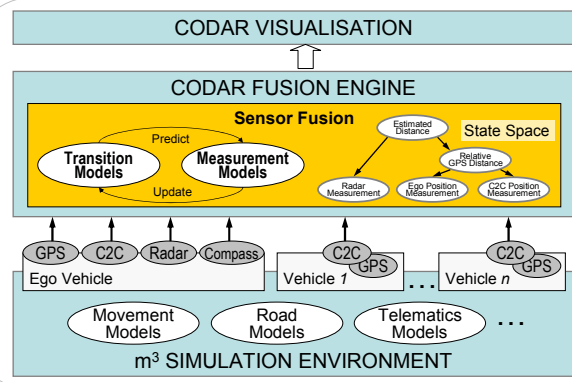

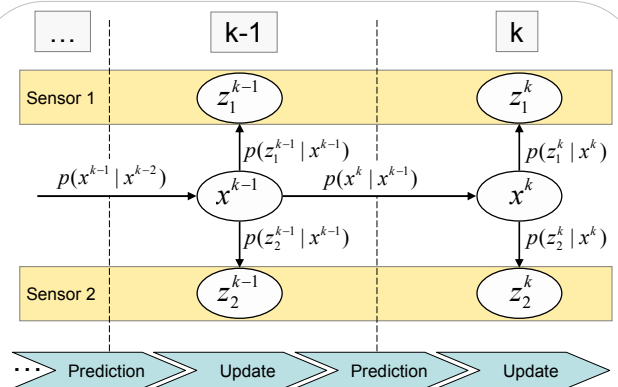

Multi-target Multi-sensor Tracking

7 Multi-sensor: e.g. Radar + V2V Communication

$>$ Multi-target: Joint Multi-target Probability Density (JMPD)

$>$ States are separated in $T$ partitions

$>$ Transition model for partitions and states

$>$ Measurement Model for partitions and states

\section{Particle Filter}

7 Sequential Monte Carlo method for Bayesian inference given inaccurate and incomplete observations

7 Recursive Prediction-Correction-Process

7 Single particle encodes a weighted hypothesis describing a possible state of the world

$>$ State of the world represents a target vehicle constellation

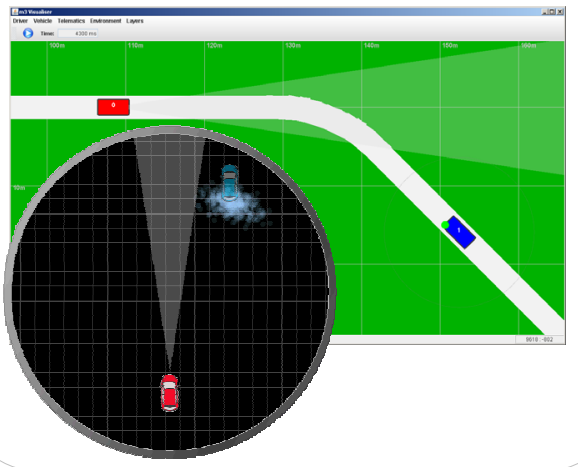

\section{Conclusions}

$>$ Exploitation of redundancy \& complementarity of information increases accuracy, reliability and robustness of situation assessment

$>$ Better foresight due to movement prediction and transmission of movement causes (e.g. brake pedal activation) instead of effects (e.g. distance decrease)

$>$ Enabler for future situation-aware driver assistance with improved safety, efficiency and comfort of driving 